\title{
Editorial: Bringing New Light to High-Pressure-Induced Phase Transitions
}

Discovering and understanding new material transformations under extremely high pressure is one of the frontiers of materials physics today. Landau theory, an approach to understanding phase transitions, is on the other hand more than half-a-century old. Can this classical, largely qualitative approach be really useful in the very contemporary context of high-pressure materials physics? As far as we understand, some in the high-pressure physics community have been rather skeptical.

We found it very remarkable, as we learned through our consideration of a paper by Tröster et al., now published as Phys. Rev. X, 4, 031010 (2014), that indeed the classical Landau-theory approach could bring much needed new light to high-pressure-induced phase transitions. We have asked an expert, Dr. Daniel Errandonea, from Universitat de Valencia, Spain, to explain why this paper is remarkable and should be read by a broad spectrum of researchers ranging from those working in the high-pressure materials physics community to those interested in statistical physics of phase transitions.

The Editors

\section{Progress in the Application of Landau Theory to High-Pressure Research: Bringing New Light to Pressure-Induced Phase Transitions \\ Daniel Errandonea, Departamento de Física Aplicada-ICMUV, Universitat de Valencia, E-46100 Burjassot, Spain}

A Commentary on:

Fully Consistent Finite-Strain Landau Theory for High-Pressure Phase Transitions

A. Tröster, W. Schranz, F. Karsai, P. Blaha

Phys. Rev. X, 4, 031010 (2014)

Phase transitions are common in Nature. They take place among equilibrium states as functions of temperature, pressure, etc. Predicting and understanding phase transitions and their effects are among the goals of condensed matter physics. A basic tool for studying phase transitions is Landau theory (LT). It was developed by Lev Landau in the 1940s, in an attempt to formulate a general theory of second-order transitions. LT describes the free energy of a system in a way that shows the nonanalyticity of a phase transition, capturing much of its physics. Its basic ingredients are the introduction of an order parameter and an expression of the relevant thermodynamic free energy as a function of the order parameter that respects the fundamental symmetry of the system. LT has been extremely useful, indeed often indispensable, in the thermodynamic description of phase transitions. One of its diverse applications is in the analysis of high-pressure-induced (HP) structural transitions in solid-state materials. Examples of applications of LT to HP transitions are ferroelastic transitions in scheelite-type oxides [1] and perovskite-type fluorides [2]. In order to give an accurate description of HP transitions, it is necessary to consider the existence of finite strains, which may become large under pressure. However, this fact has been neglected in the past: Pressure-induced strains are routinely treated as being infinitesimal, a hypothesis that is not always valid upon compression.

The article by Tröster et al. [3] addresses this fundamental shortcoming by introducing a new theory much needed by the HP physics research where high-quality experimental data await analysis and interpretation. It presents a highly nontrivial extension of LT to finite strains that treats fully the structural nonlinearities generated by high pressure. By applying their theory to $\mathrm{SrTiO}_{3}$, a model perovskite, Tröster et al. demonstrate clearly, pedagogically, and compellingly the need and power of applying finite-strain LT to modern high-accuracy HP experiments: They have provided a precise description of the high-pressure cubic-tetragonal transition of $\mathrm{SrTiO}_{3}$, which the classical infinitesimal-strain Landau theories fail to do, and they have predicted novel phenomena that are accessible to experimental verification. Finally, the paper serves as an important basis for future theoretical work on the understanding of materials properties. 
In summary, the article represents a breakthrough from previous studies of HP-induced structural transitions. The reported results on the application of the theory are remarkable and will be quite useful to HP scientists in their analysis and understanding of state-of-the-art highpressure experiments. The presentation of the theory in its functional form will facilitate its implementation in, or adaptation to, practical cases, even for researchers unfamiliar with nonlinear mechanical aspects of physics. I believe that these qualities will enable the paper to generate a long-term impact. I recommend that scientists working in the area of high-pressure materials physics and readers of PRX interested in phase transitions get fully acquainted with it.

About the Commentary author:

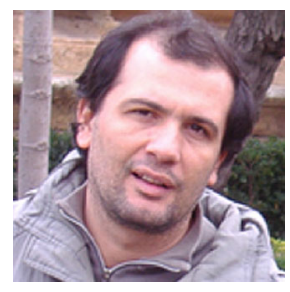

Daniel Errandonea was born in Argentina. He is a professor at the Department of Applied Physics of the University of Valencia, Spain. His research interest is physical properties of materials under high-pressure and high-temperature conditions. He won the Alvin Van Valkenburg Award among others for his scientific contributions.

[1] D. Errandonea, Europhys. Lett. 77, 56001 (2007).

[2] M. Guennou, P. Bouvier, G. Garbarino, J. Kreisel, and E. K. H. Salje, J. Phys. Condens. Matter 23, 485901 (2011).

[3] A. Tröster, W. Schranz, F. Karsai, and P. Blaha, Phys. Rev. X 4, 031010 (2014).

Published 17 July 2014

DOI: 10.1103/PhysRevX.4.030001 